\title{
Guest-Editorial
}

\section{Tissue Engineering for Orthopaedic Applications}

The European Society for Engineering and Medicine (ESEM) hosted a Tissue Engineering for Orthopaedic Applications Symposium in Prague, Czech Republic on November 21st, 2005 at the 3rd European Medical \& Biological Engineering Conference, EMBEC'05.

ESEM seeks to build a bridge between engineering and medicine and this is essential in tissue engineering, which involves the application of principles and methods of engineering and life sciences toward fundamental understanding of structure-function relationships in normal and pathological mammalian tissues and the development of biological substitutes to restore, maintain or improve tissue function. More information on ESEM can be found at: www.esem.org.

Tissue lost due to disease, trauma or excision may be replaced by autografts, from the same patient, allografts, from a human donor, xenografts, from another species, or by tissue engineered constructs. Auto- and allograft supplies are limited, while allo-and xenografts pose infection risks, hence the increasing interest in tissue engineering which uses porous scaffolds seeded with cells. These seeded scaffolds may be implanted directly into the injured site and regeneration of tissues or organs induced in vivo. Alternatively, the cell scaffold constructs may be subjected to mechanical stimuli in bioreactors, or stimulated by chemical growth factors, and cultured in vitro to synthesise tissues which are then implanted.

Orthopaedic applications pose a particular challenge as, in most cases, the tissue engineered construct must have sufficient mechanical strength to function in situ from the time of implantation to the completion of the remodelling process. Thus the construct must be strong enough to allow handling during surgery and have mechanical properties consistent with the anatomical site into which it is to be implanted. Producing scaffolds with adequate mechanical properties is one of the great challenges in attempting to engineer bone or cartilage. Furthermore, healing rates vary - in young patients, fractures normally heal to the point of weight-bearing in about twelve weeks, with complete mechanical integrity not returning until approximately one year, but, in the elderly, the rate of repair slows down.

This Special Issue of Technology and Healthcare opens with a paper by O'Brien et al., which focusses on the material properties of scaffolds. They characterised the permeability and fluid mobility of collagenglycosaminoglycan scaffolds as a function of pore size and compressive strain using both experimental and mathematical modelling techniques. The results of both analyses revealed that scaffold permeability increases with increasing pore size and decreases with increasing compressive strain. The correlation between predicted and measured scaffold permeability suggests that cellular solids modelling techniques can be utilised to predict scaffold permeability in a variety of pore microstructures under different physiological loading conditions.

The second paper by Farrell et al. characterised the differentiation capacity of adult mesenchymal stem cells (MSCs), cultured on plastic coverslips in monolayer, when seeded on these collagenglycosaminoglycan scaffolds in the presence of a standard osteoinductive cytokine cocktail. Cells were 
cultured for 3, 7, 14 and 21 days and several markers of osteogenesis were analysed. While the initial response of cells in 3-D seemed to exceed those cultured in 2-D, later markers showed that osteogenic differentiation of MSCs took longer in the 3-D environment. Significantly however, it was shown that complete scaffold mineralisation occurred by 21 days, demonstrating the potential of MSC-seeded collagen GAG scaffolds for bone tissue engineering.

The third paper by Fassina et al. looks at the effects of electromagnetic stimulation on human osteoblasts seeded onto porous polyurethane. In comparison with control conditions, electromagnetic stimulation caused higher cell proliferation, higher surface coating with decorin and type-I collagen, and increased calcium deposition demonstrating that the superficially modified biomaterial might be used in clinical applications as an osteoinductive implant for bone repair. This earned Lorenzo Fassina the ESEM prize for the best conference paper in the area of Cellular, Molecular and Tissue Engineering.

In the fourth paper, Pilliar et al. use a porous calcium polyphosphate (CPP) and chondrocytes in cell culture to develop a construct in which the pores anchor the cartilage. These biphasic constructs were implanted in sheep femoral condyle sites and left for short-term periods ( 3 to 4 months) or longer periods (9 months). Implant fixation within the condyle sites was achieved through bone ingrowth into the inferior CPP pores. The properties and characteristics of the in vitro-formed, short- and long-term implanted tissues were compared and the results indicated that such implants might be useful for repairing small subchondral defects.

Appropriately, the final paper in this Special Issue, by Dickson et al., reviews recent developments in the field of tissue engineering for orthopaedic applications. The authors note that medical implants which encourage natural tissue regeneration are more desirable than metallic implants, which may require a second procedure to remove them. Numerous polymeric materials, from natural and artificial sources, are under investigation as substitutes for skeletal tissue. For bone regeneration, cells (obtained mainly from bone marrow aspirate or as primary cell outgrowths from bone biopsies) can be combined with biodegradable polymeric materials and/or ceramics and absorbed growth factors so that osteoinduction is facilitated together with osteoconduction through the creation of bioactive rather than bioinert scaffold constructs. Relatively rapid biodegradation enables advantageous filling with natural tissue while loss of polymer strength before mass is disadvantageous. Innovative solutions are required to address this and other issues such as the biocompatibility of material surfaces and the use of appropriate scaffold topography and porosity to influence bone cell gene expression.

The challenge of tissue engineering is to mimic what happens in nature. To date, the highest rates of success have been achieved in the areas of skin and bladder regeneration where tissue-engineered material has been used successfully in the clinic. In orthopaedics, the greatest successes to date have been in the area of cartilage generation in vitro where some tissues have been implanted in patients. However, a fully functional, biocompatible, mechanically competent, vascularised tissue-engineered substitute for bone or cartilage remains to be developed. As more sophisticated biomaterials and bioreactors are developed, and as we learn more of the cell signalling mechanisms involved in tissue development, we will get closer to achieving these aims. Close collaboration between engineering and medicine will speed the process.

T. Clive Lee and Fergal J. O'Brien Guest Editors Royal College of Surgeons in Ireland \& Trinity College Dublin Ireland 